\title{
Digital Graphic Design Systems for Sustainability in Teaching Aids for the Illiterate
}

\author{
Sally Ali Abdelhalem zaitoun ${ }^{a}$ \\ ${ }^{a}$ Alexandria, 43Amr Al Ngomai, Sidi Beshr Bahri, Alexandria Postcode 21611,Egypt
}

\begin{abstract}
The research explains and analyzes the most important digital methods that depend on graphic design of elements and icons. These help illiterate people comprehend shapes, words, letters, and numbers, which greatly affect the process of understanding the matrix and the information available through the applications which are used. These systems allow diversification and distribution of elements and spaces using colors and consider how the recipient perceives this information. Further, the research sheds light on the role of electronic games and other applications that have become an integral part of the information design process in a graphic form that follows modern rules and regulations in this field. The research presents foundations of designing educational applications, some samples of literacy applications and displaying samples of speech recognition and handwriting applications, the main requirements for designing an electronic application. Requirements. The research also identifies the problem in the form of questions, and the research continued the explanation to reach its goals, ending with several findings and recommendations.
\end{abstract}

\section{KEYWORDS}

Educational applications -electronic game -illiterate people.

\section{INTRODUCTION:}

ICT has provided valuable educational assistance and has revolutionized education. This has forced educators to involve ICT in education to improve the effectiveness of teaching and learning outcomes. Recently, educators have turned their attention to 3D virtual environments because they have great potential to simulate near-real-life experiences in a cost-effective way that makes them suitable for learning from traditional binary applications. (Inayat ur-Rehman \& Azra Shamim, 2016) 
Technology for adults with low levels of literacy has facilitated effective communication with mobile application, and interaction with mobile technology through touch screens is a simple and easy mechanism for adults with limited literacy skills. Continuous learning is essential for literacy-free people, adult literacy programs and associated program applications play an effective role in raising literacy levels and supporting their day-to-day tasks in education. (Lumsden،J\& Leung, R\& Fritz،J, 2005)

\section{MATERIALS AND METHODS}

\subsection{Classification of Learning Applications:}

"Learning applications" can be classified in several ways. One classification may focus on "educational design" criteria or addressing "learning goals" for a particular application. Another classification system may focus on motivational areas through "motivation," "reward systems" or the amount of "information and entertainment." Applications have been classified into three nonexclusive categories: Game format, Interactive eBooks and Open-Ended (Fig. 1).

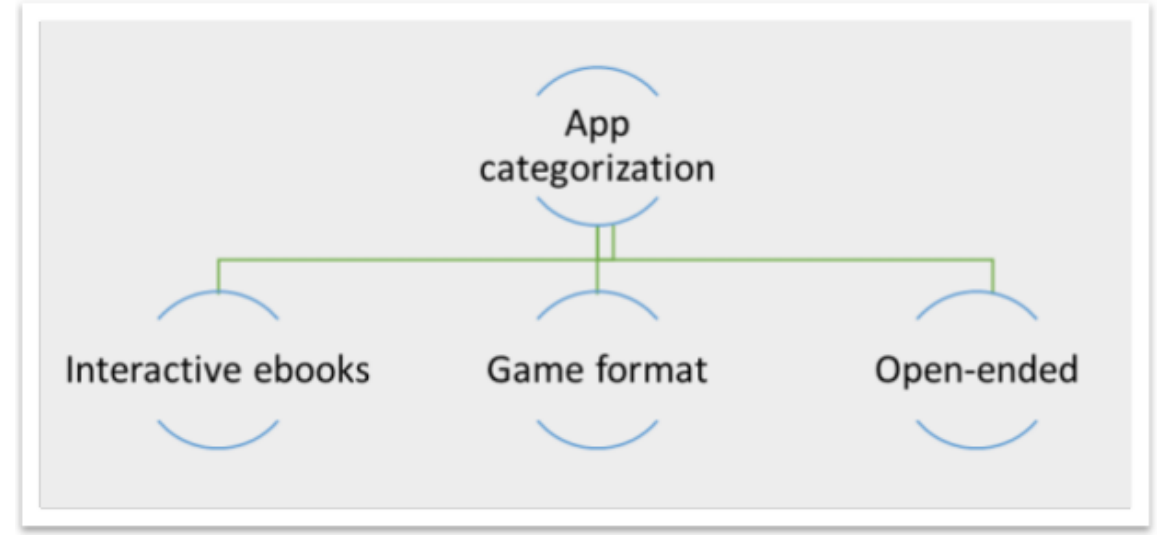

Fig. 1- shows Classification of mobile applications.

Interactive e-books are applications that combine text, audio, narrative, images, animation, and video. Multimedia features that enable users to participate in interactive data exploration are used extensively. Applications of the game (Game format) are usually combined with interactive 
features and educational content, either in the form of memory activities (such as matching or hiding numbers, characters, and images). Open applications (Open-Ended) contain a variety of content without the game's target. (Stamatios Papadakis \& Michail Kalogiannakis,2017)

\subsection{Smartphones as Mobile Learning Environments:}

Smartphones, such as mobile learning environments, provide many learning possibilities and opportunities. Smartphone features include cameras, text features, audio and video content, access to learning applications, recording possibilities, global positioning systems (GPS), Internet access, e-mail, word processing, readers, calculators, and many others. Smartphones can offer unique educational opportunities, as smartphones run on mobile operating systems such as Google Android, Apple IOS and Nokia Symbian. It can run many free and paid applications, converting dedicated mobile phones into powerful and mobile personal computers. Smartphones are useful in creating educational spaces for people with low literacy or illiterate people. Smartphones provide support for reading and mathematics from applications such as Khan Academy and Virtual Reality Trains. It also promotes literacy skills through the provision of dictionary services. It proves to be a catalyst for learners when integrating technologies, such as GPS or YouTube. (Tia Wilkinso, 2020)

\subsection{Electronic games:}

E-games have spread widely to young and old. It is good to use them for fun education. This educational method is centered around the learner. It has effective states instead of the role of the recipient. It is one of the most important means of education for its quality educational materials. It has a specific educational purpose. And with our technological revolution and the spread of computers and laptops and various gaming devices like PlayStation, Xbox, Gameboy, Wii, and tablets like Galaxy Tab, iPad, iPod, and smartphones like iPhone, Blackberry, Galaxy. 
Electronic games became widespread and were used by young and old, so it was necessary to use them in education to achieve educational goals. Educational games can also be used in all subjects and with all levels of learning, dental and cognitive, and helping those who are free from illiteracy to continue learning until they are reminded of what they have studied before in a fun and meaningful way.

\subsubsection{The Concept of Educational Electronic Games:}

A set of interrelated activities and processes performed by a learner on a computer, a smartphone, or a tablet device, to achieve a specific learning goal in a fun setting, a type of learning centered around a learner and allowing him/her to interact within an educational environment, usually with educational games in a form that attracts the learner and makes him/her not leave the game without achieving the desired goal.

\section{3.2. Educational Electronic Game Features:}

Electronic games have many features, including:

- The use of audio and visual effects, which make learning more effective and sustainable.

- The tendency of learners to play, increasing their motivation for learning, especially new subjects.

- The possibility of using them effectively in teaching different subjects.

- Development of visual attention and motor sensory consistency, as it requires attention to and rapid response to several effects in different areas of the screen.

- The division of information into small parts that require a response and provide rapid feedback and prompts the learner to continue playing and reaching the desired target.

- Repetition of educational computer games at any time that ensures repeated learning. 


\subsubsection{Electronic Game Design Standards:}

For the successful design of the electronic game, there are some criteria, including:

- Achieving educational and educational objectives and supporting the curriculum.

- The instructions are clear and contain the tribal education required for each skill.

- The game is suited to the pupil's cognitive potential and contains elements of suspense.

- To step from easy to difficult and to be easy to use and to provide immediate weighted feeding.

- Its content should be focused on the interests and tendencies of learners and should satisfy their biological needs and demands. (https://esrc.org.lb/article.php?id=4399\&cid=248\&catidval=0)

\subsection{Cognitive and Knowledge Aspects of Mobile Applications:}

\subsubsection{Cognitive Aspect: CAPITAL Words application for adult learners shows usability}

It is an educational application (Android) to help literate adults improve their voice awareness. This suggests that if users are given a small amount of guidance at first, there is a high likelihood that they will be willing and able to continue using the application independently to improve their reading and writing skills. There are design options that are effective in transmitting educational content in a simple-text interface.

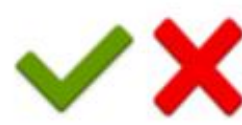

(a)

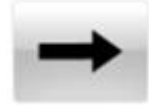

(b)

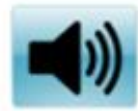

(c)

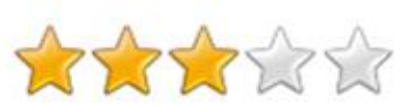

(d)

Fig. 2- Shows (a) a Check Sign to indicate right or wrong; (b) Right-handed Arrows Showing Advanced screens; (c) Codes Headphones to Denote Operable Sound; and (d) 5 Stars to Represent the Result.

\section{- Iconography:}




\section{INTERNATIONAL JOURNAL OF}

Almost essentially depends on images to represent ideas transmitted by the typical interface. We use existing icons where possible to convey high-level concepts: For example, right-facing arrows are used to represent forward progress, and questions' answers are classified with correct or error selection marks to show their validity or error.

\section{- Linearity:}

With a directed linear design, we organize screens in sequential order to guide users through tasks. We reduce user input to a semi-permanent " Continue or next" button that moves to the next screen. Learners can simply move forward screen by screen to access and complete their educational materials, after which the next step "forward" will return them to the beginning of the next set of materials.

This " previous" mobility button also allows flexibility to offer optional options. Each course lock is cancelled in serial order, leaving all pre-completed classes available for re-enrolment at any time.

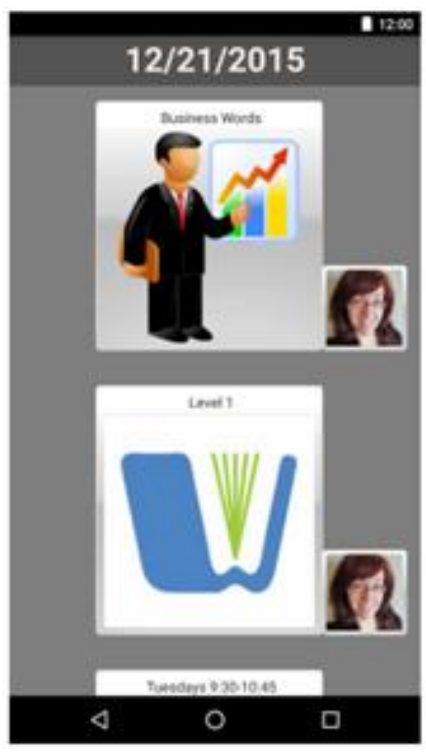

(a)

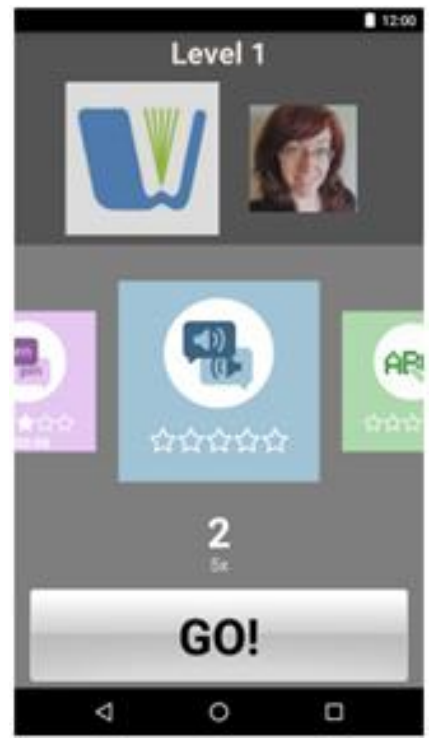

(b)

Fig. 3- shows (a) a pass-through list of course cards, each with a unique image and a picture of the instructor; and (b) the same image/image displayed at the top of the session page. 


\section{- Consistency}

Reducing the cognitive load of a learner when moving through an application is a primary goal. To that end, we sacrifice modern minimalist aesthetics in favor of a highly varied design, using consistent and distinct methods to clearly indicate the functions and relationships of the elements.

\section{- Pattern}

Applying a "shiny" gradient pattern to all interactive elements to help them distinguish themselves from their static surroundings. This allows learners to easily identify button components that can move them to a new screen, run an audio file, send an answer, etc. This mode can be applied to all colors and formats to complement other design options. Colors Each exercise type in the application contains an associated color that is used for all relevant components (background, buttons and card faces). These colors are for workout items and are not used anywhere else in the application. The high-profile use of color clearly knows what kind of questions it will answer, which is useful for distinguishing between types of exercises without written claims. Shapes and using a rectangular format to represent those elements that are "modules" with other elements in them (such as training courses and lessons). Using the consistent shapes and sizes of each card, we visually associate it as: For example, each training course card differs only through its images, showing that it is structurally identical but contains unique content. (Jennifer Hill\& Rahul Simha, 2016)

\subsubsection{Knowledge Aspect: Creating a learning environment for illiterate spelling:}

\subsubsection{Application of speech recognition and handwriting:}

The hallmark of the application is the integration of speech recognition and handwriting, as well as audio-visual means as in (figure 4) key elements:

\section{- Presentation}




\section{INTERNATIONAL JOURNAL OF}

First, the symbol appears in its known form. After that, the short vowel sounds of the symbol are displayed and their sound is displayed, along with an animated display of the examples, which the machine also reads out loud. The exact addition that can be useful is to move the set of customary characters to form connected symbols.

\section{- Primary Reading and Writing}

For all character models, the learner is asked to pronounce the sound after the device. After that, the student is asked to write each of these forms, following the machine's animation of the writing movement.

\section{- Contextual Listening}

A general paragraph with examples of acquired symbols is presented and read aloud by the device. The symbols learned are distinguished to emphasize their use in the context of a larger text.

\section{- Exercises}

After presentation, initial reading and writing, and contextual listening to a set of symbols, the lesson offers exercises to evaluate the student. Exercises do not provide guidance in primary reading and writing but use the same recognition engines. They either ask visually, and request spoken responses, or they demand voice and request written replies. Some gaming exercises are organized to engage learners more effectively.

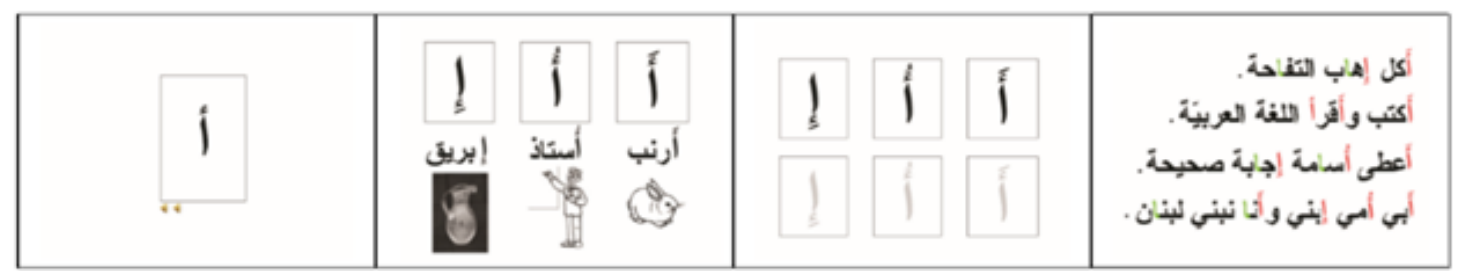


(a)

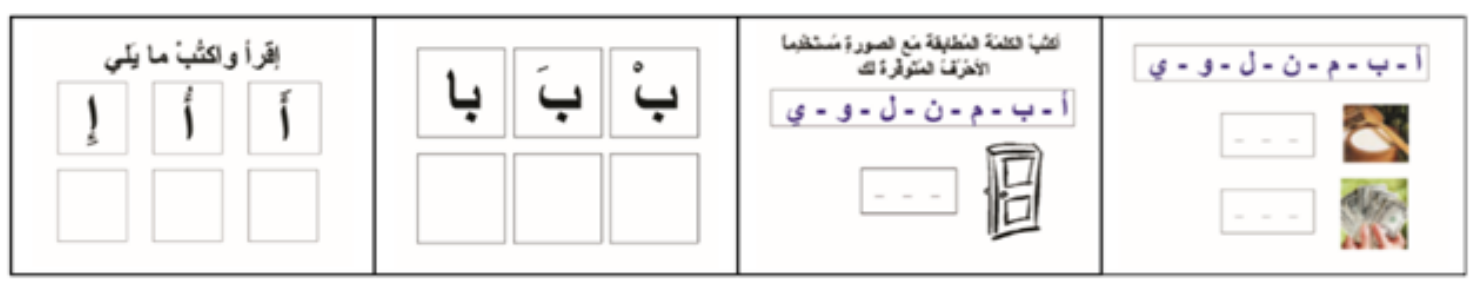

(e)

Fig.4- illustrates a typical interface offering (a) presentation, (b) primary reading, (c) writing, (d) contextual listening, and (e) exercises.

\subsubsection{Handwriting Recognition:}

For handwriting in fig.5, the Multiple Gestures Recognition Tool has been developed, which uses the recognition of individual gestures through multi-layered neural networks to feed forward, but the input is explained by comparing relative distances, sizes and the tendency of individual gestures, this component is implemented directly in Microsoft Visual C++.

Handwriting is obtained through a touch or pen screen on a tablet computer. When a letter, part, word, or sentence is inserted, the entry is specified as a set of distinct connected gestures. Individual gestures correspond to parts related to Arabic handwriting, such as different connected forms. Each nod is initially represented as a ranked set of point coordinates, the node is represented at the angle of the vector difference for consecutive points.

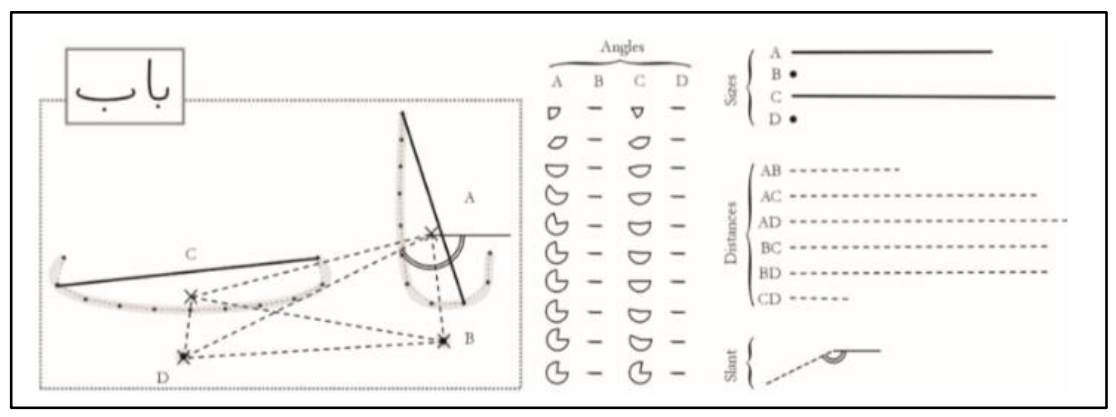

Fig.5- shows a complete representation of handwritten entries. 


\subsubsection{Speech and Handwriting Engine Test Interface:}

Fig. 6 shows a test interface for testing speech engines and handwriting. Some of the elements we are working on to improve the learning experience are as follows:

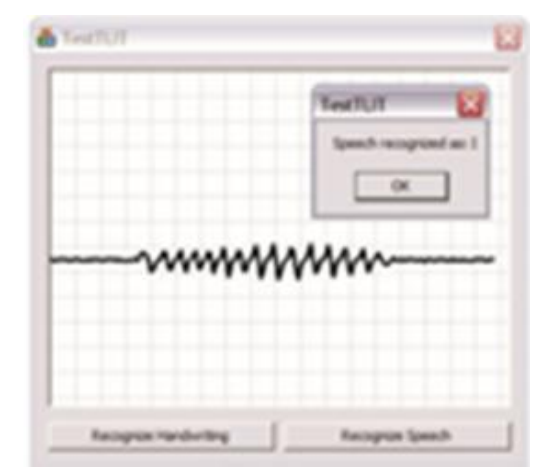

(a)

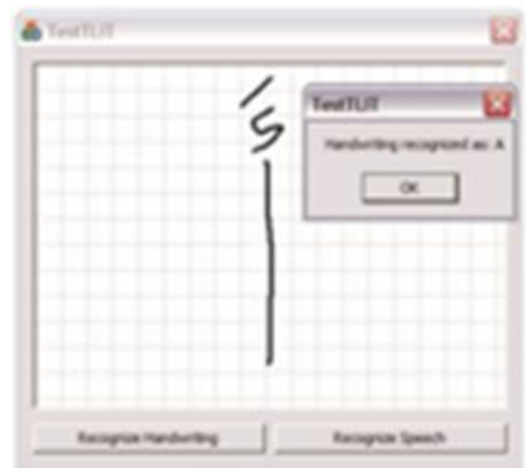

(b)

Fig.6- Illustrates the Technical Component Test Interface, Showing (a) Speech and (b) Handwriting Recognition.

- Direct computer literacy :The program includes components that train users in computer literacy fundamentals.

- Daily scenarios :An individual can imagine integrating different real scenarios as part of a lesson material. For example, visiting a hospital or preparing an airport can be useful scenarios for teaching numbers.

- Smart assessment and adaptation :Technology can be used to promote learning by means that go beyond speech recognition and handwriting. For example, the system can use the learner's performance log for exercises and training exams to maintain the user's identification file.

- Instructions :To make the program accessible to ages, it is necessary to pronounce instructions out loud. The inclusion of textual versions of these instructions, and their visibility, can provide the learner with more exposure to the material learned.

- Attractive graphics :The more attractive the subject is, the more attractive it becomes for the learner.

- Independent Tool :Speech and handwriting can be integrated with the system of organized lessons in more creative ways. For example, the "dictionary" feature, where 
small vocabulary can be queried by voice, to get a spelling and a word illustration (Mohamad Adnan Al-Alaoui, Mesrob I \& Ohannessian, Ghinwa F, 2007).

\subsection{Applications that Illustrate the Learning of Arithmetic: Virtual Learning Environment for Illiterate Adults}

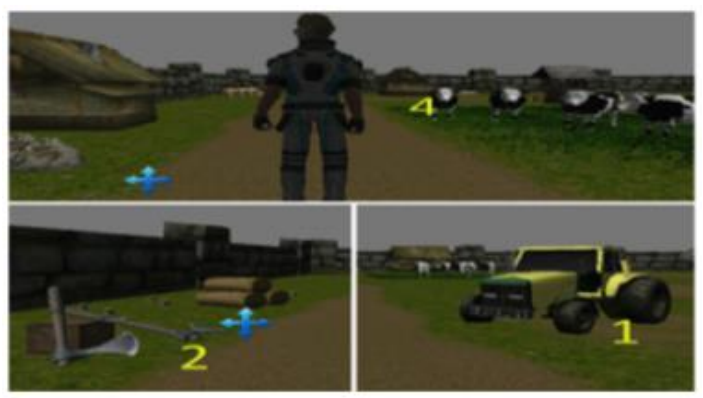

Fig.7- illustrates farmers' virtual learning environment

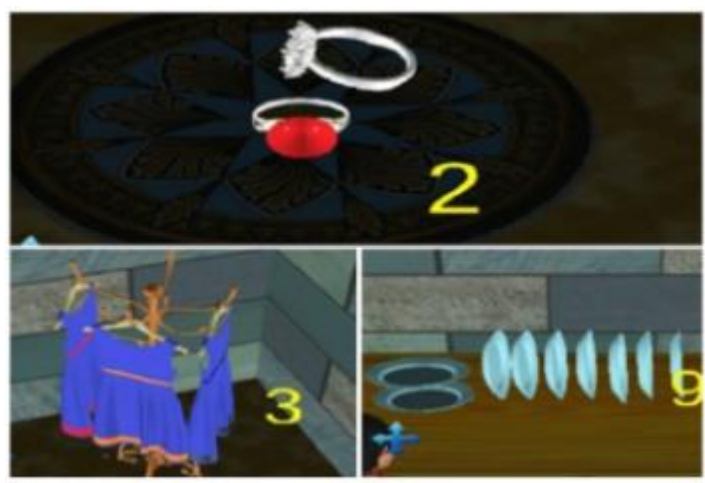

Fig. 8- illustrates the virtual learning environment of women showing the teaching of arithmetic.

In this educational application, it has three types of environment: the general environment, the farmers' environment, and the women's environment. They were designed by specialists based on a sense of belonging. The user interface for such environments was developed using a Greek 3D engine. Fig.7 and 8 illustrate the proposed environment for farmers and women (Inayat urRehman\& Azra Shamim, 2016). 


\section{INTERNATIONAL JOURNAL OF}

\subsection{Applications that Illustrate Punctuation}

\subsubsection{Punctuation App.}

Punctuation marks app is designed to teach when to use a period at the end of a sentence, a question mark, and where to use quotes (about phrases to show the words that are being said, as well as about titles for stories, songs, books, and movies).

Punctuation application does not start with any type of list, but immediately begins with an introductory explanation as the period is dragged with a hand to the end of the sentence. From this screen (Fig. 9), the user is then left to drag the remainder to the end of the next sentence.

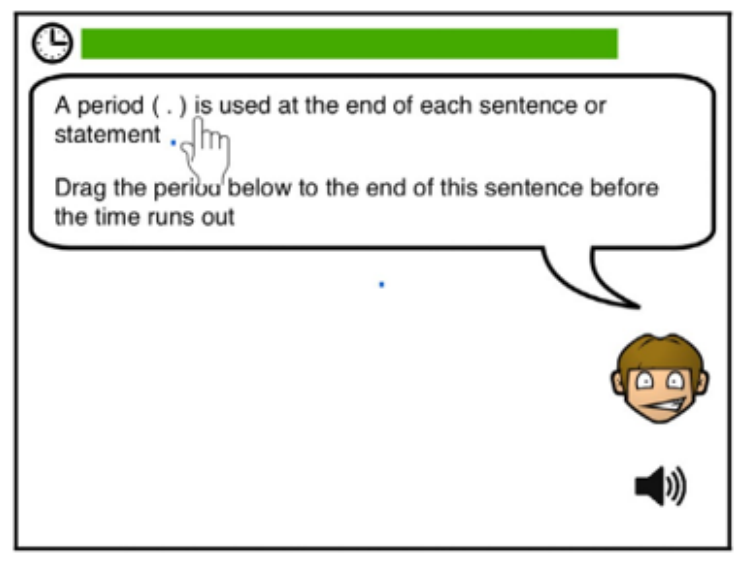

Fig.9- shows an introduction to applying punctuation.

A horizontal bar at the top of the screen next to the clock graphic tells the user how much time is left. The bar will turn yellow when only $25 \%$ of the allotted time remains. If the user completes this step, as shown in Figure 10, they will initiate a virtual "tour of Italy" which simultaneously attempts to teach the user the correct use of question marks and quotes. 


\section{INTERNATIONAL JOURNAL OF \\ DESIGN AND FASHION STUDIES}

VOLUME 4, ISSUE 1, 2021, $160-182$.

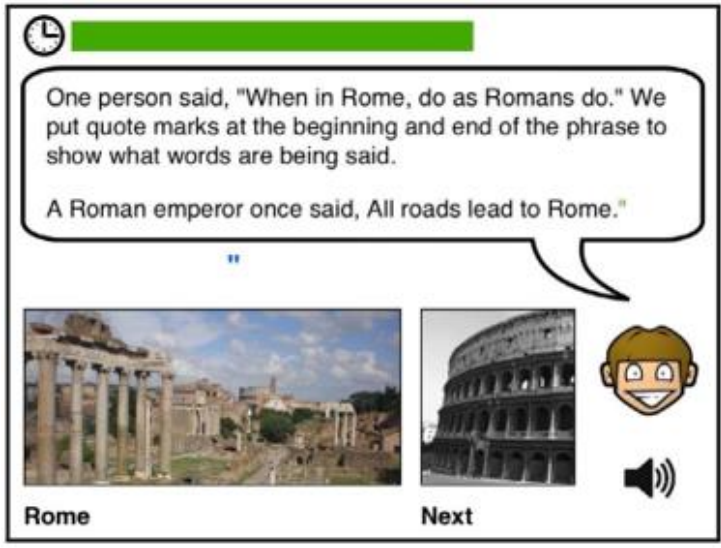

Fig. 10- shows a tour of applying punctuation.

Each screen presents the user with a series of sentences requiring the appropriate punctuation to be dragged to the correct places before the timer runs out to advance to the next screen. The user receives an explanation of how to properly use each punctuation mark in the first punctuation screen. If the user drags the alert flag to the correct position, the approval sound is played. If the user drags a punctuation mark to the wrong position, it returns to its original position under the text. If the user does not complete the task in time, the correct answer will be presented to the user as an audio explanation as to why that answer is correct. The user then duplicates the same screen until they drag the correct punctuation marks to the correct positions. The numbering application screens get more difficult with the passage of time, as the user is initially given one or two punctuation marks only to the correct position before giving it three and then only four as shown in Fig. 11. 


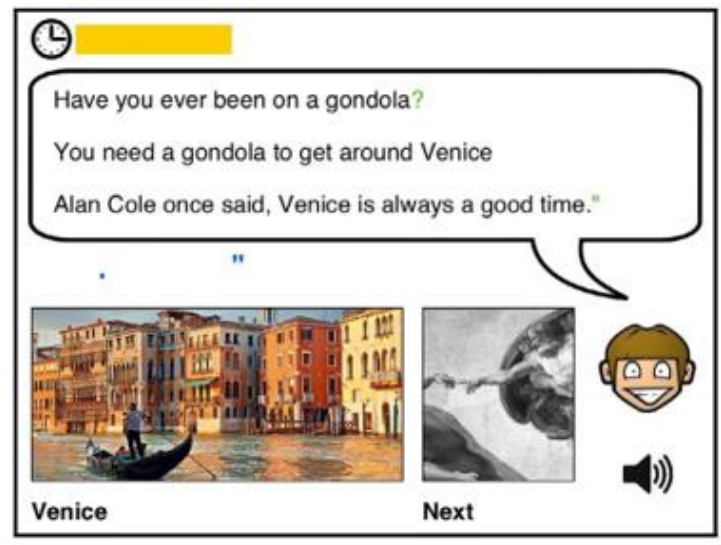

Fig.11- shows the increase in difficulty.

There are 10 screens in the Punctuation app. All text on each screen is also presented to the user as audio. When the user completes the original screen, they are given a farewell to congratulations by the character who guided them during the tour in the lower right side of the screen. The images of Italy appearing throughout the app were intended to provide an aesthetically pleasing reward for reaching each screen, with a gray image of the next screen providing some motivation for figuring out the next step.

\subsubsection{Comma App.}

Comma implementation is like the practice feature for applying punctuation, but with some differences. The app begins with an illustration where the image of a hand pulls a comma to the correct position in a sentence. The user is then given a cycle of aging in which he must pull the comma to the correct position. If the user drags the comma to the correct position, they will receive an approval vote and a green check mark as shown in Fig.12. 


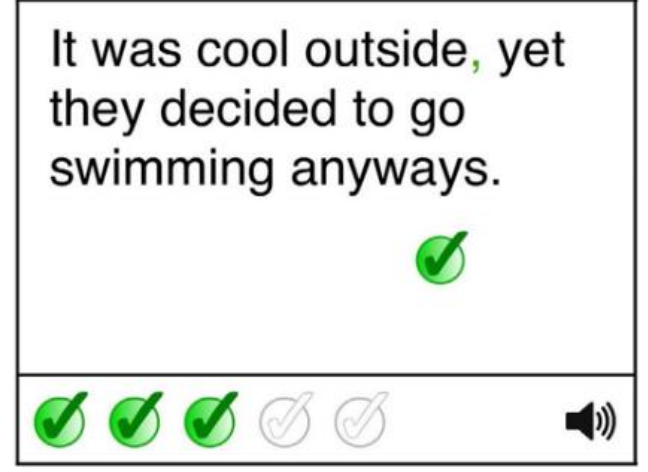

Fig. 12- shows a correct answer for applying a comma.

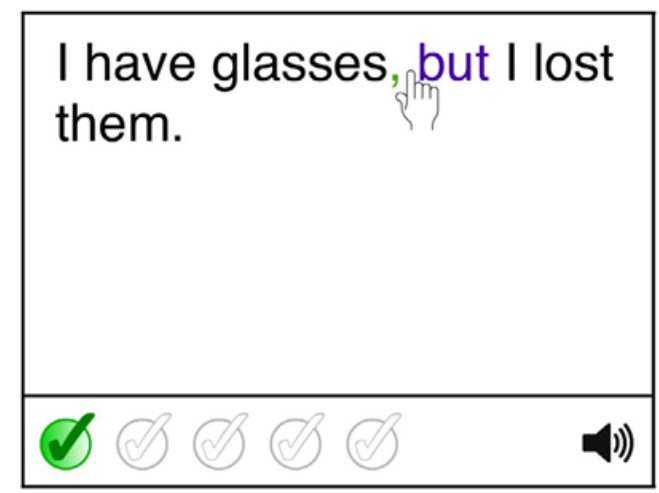

Fig. 13- illustrates an explanation of a correct answer

If the user drags the comma to an incorrect position, as shown in Fig.13, the correct answer is explained using the voice. From here the user moves to the next sentence which was not completed correctly.

Remaining sentences remain in the course until they are properly completed. A series of gray checkmarks in the lower left corner of the screen turn into green checkmarks as the user correctly completes each sentence. When the user completes all the sentences correctly, they are given a gold checkmark and the application is completed. (Kevin Browne, Christopher Anand, 2014) 


\section{RESULTS AND DISCUSSION:}

\subsection{Design problems for some educational applications}

- In general, voice quality was poor in most applications, both during play and in note messages. Similarly, quality was very low in image use and generally multimedia use in most applications.

- Although many applications use interactive active hotspots, it has not worked. Also in some applications, there was no clear distinction between the different interactive elements of the application and its background. It was difficult for the user to distinguish what components of the application were part of the background data or formed interactive elements associated with the game story (fig. 14-a).

- In most applications, the user interface was not well designed or easy to use. It is easy and good when the list is hierarchically arranged. In some applications, lists were not logically arranged, and there was often no correlation between the application's control elements (menus and buttons) and the screen's transitions. Also, in many applications, the user had to choose elements that were either too small or were placed close to each other. Which led to the wrong choices. The touch screen, with a size of $0.32 \mathrm{~cm}$ ( 0.125 inches) or less, is very small for both adults and children (Fig. 14-b, c).

- In some applications, the screen was crowded with buttons, characters, mobility shares, etc., which complicated processing.
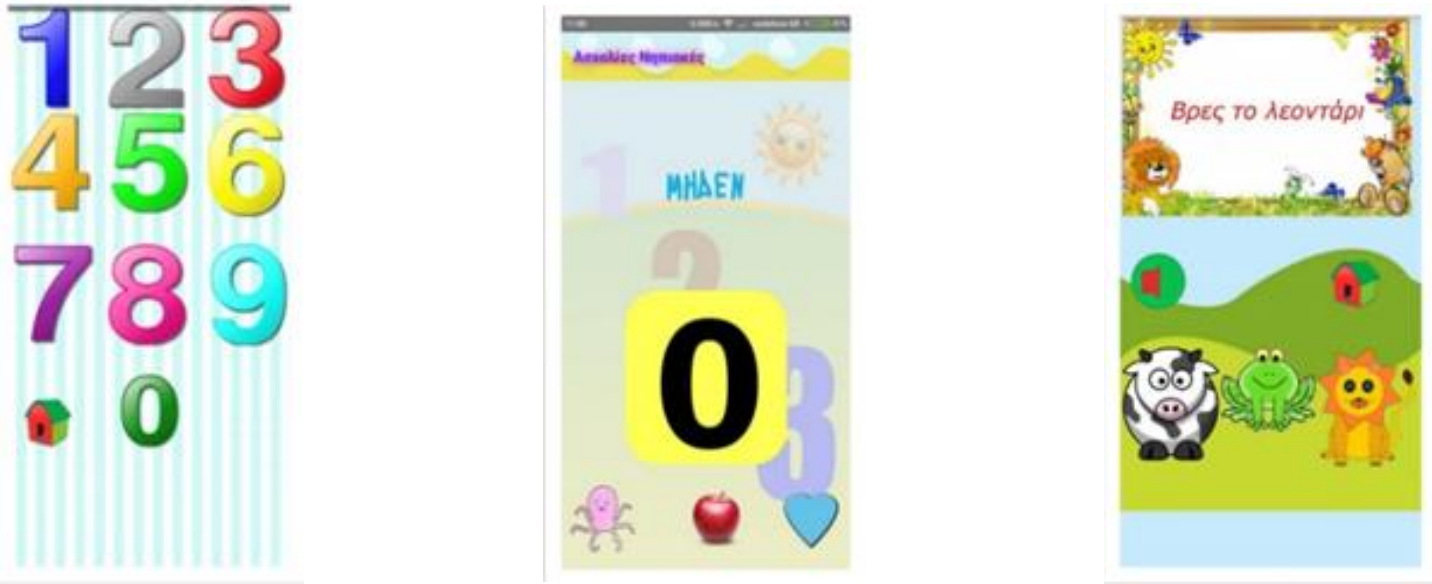
Fig. 14- illustrates (a, b, c) design problems

- Many applications did not allow the user to switch between the application screens or return to the main menu screen using the application control elements. Sometimes the stop button was absent. In some cases, the only option for the user was to use the key buttons at the front of the device. This caused confusion among the user (fig. 15).

- At this stage, it should be noted that the use of the "home page button" does not end the application. The application continues to run in the background, draining the resources of the mobile device. The presence of this type of application on a mobile device causes problems of stability, slow response time and low user satisfaction.

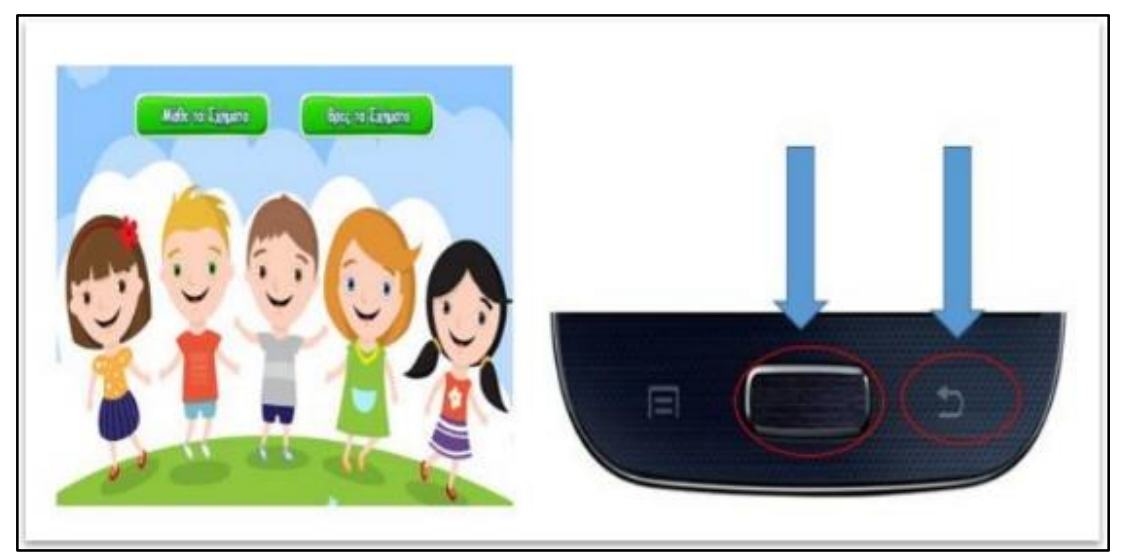

Fig. 15- shows an application without a termination button. The user had to use the device's controls.

- Most applications focus on literacy (70\%) and mathematics (30\%). Literacy applications aimed at teaching the alphabet (identifying the letters and sounds of spoken alphabets), while mathematics applications aimed at teaching the basics of numbers, applications did not attempt to develop a higher level of reading skills such as reading accuracy, fluency, and spelling or self-expression skills. There have been no applications focused on developing general cognitive skills such as screening, classification, or a sense of numbers as a group. 
- Most applications had interactive content but were closed, i.e., activities that could not be modified or extended by the user (16-a, b, c format). Applications tend to assess the level of knowledge that exists only in advance and have not attempted to teach new concepts.

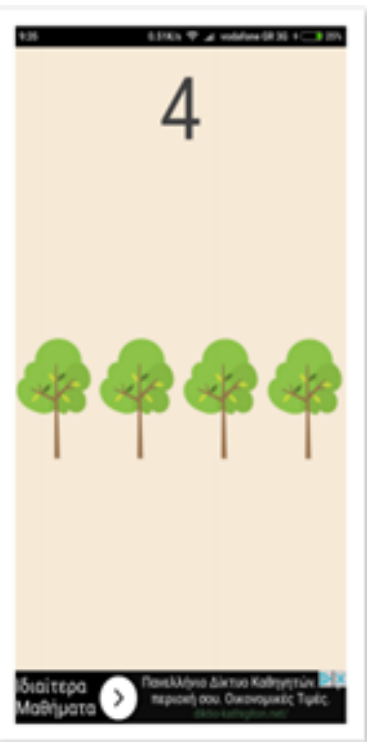

(a)

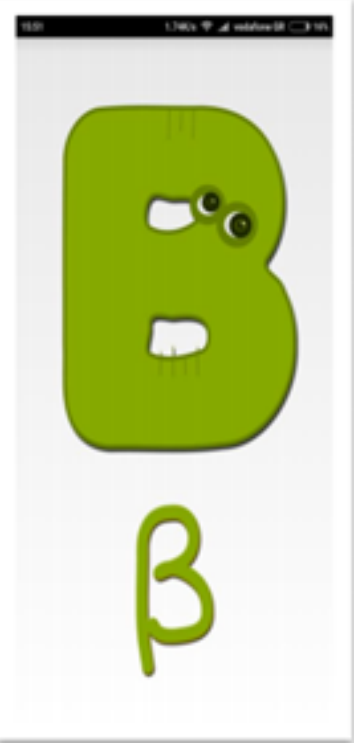

(b)

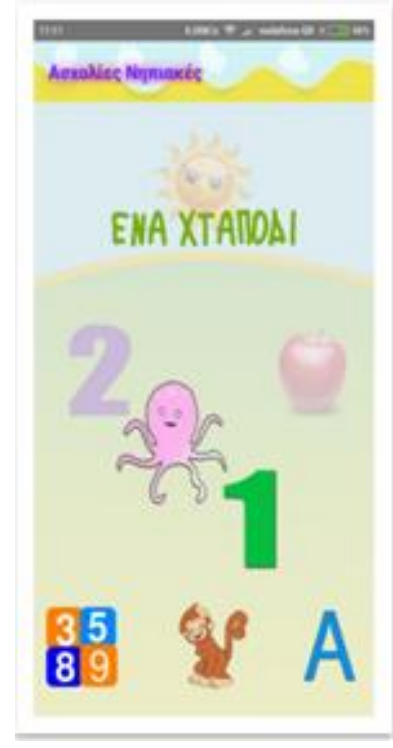

(c)

Fig. 16- shows (a, b and c) examples of applications with closed content.

- Most behavioral-type applications. It guided users to solve problems through "trial and error" questions rather than through effective and meaningful learning experiences. The user often found the correct answer only after all the answers had been exhausted. For example, if the user does not know the answer to question " $1+2$," he or she can tell the right through trial and error.

- In many applications, whenever the user chooses to handle the same application at different times, the content of the application is identical each time. The random distribution feature was absent.

- Some applications did not provide any guidance on how to use them. Many applications provided guidance that was incompatible with the user category. An example of an 
application, although aimed at pre-school-age children, relies on the use of pre-written text in the downgraded lists or on the main menu screen, rather than using element graphs (see fig. 17).

- Many applications did not provide a "palm rest" area, which is an area in the lower part of the application that is free of buttons and interactive elements, and which can lead to the operation of application functions. The lack of a "hand rest" area becomes more problematic in user-targeted applications. In properly designed applications, this area is automatically disabled or hidden - if it contains other graphical user interface buttons and elements - so as not to impede application functions (fig. 17-c).

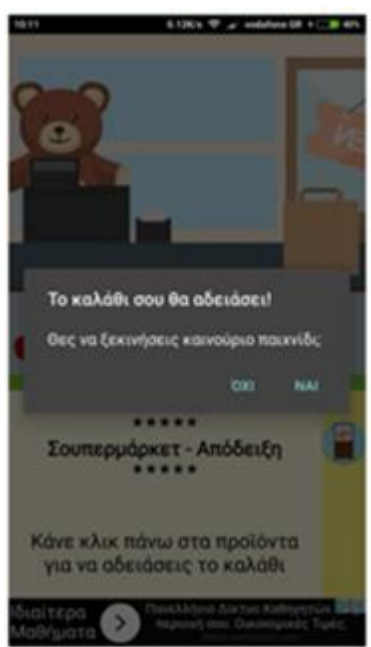

(a)

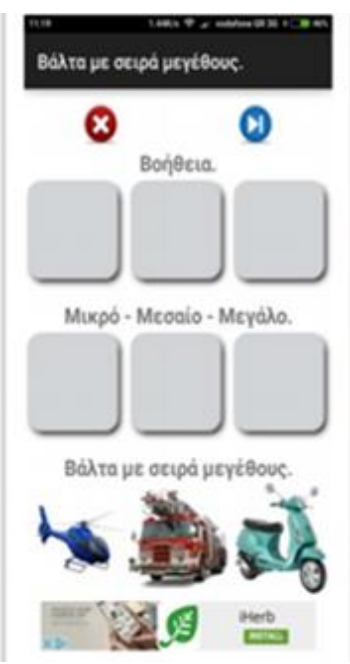

(b)

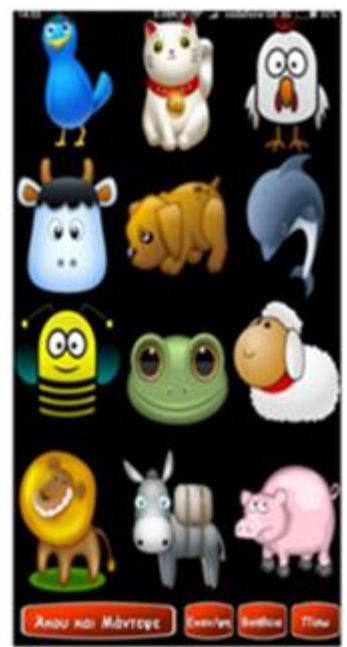

(c)

Fig. 17- illustrates (a, b, and c) Applications with problematic functions.

- The presence of advertising has been particularly intense in many applications. Occupy a large screen space, it distracts and confuses the user. (Fig. 18- A, B, C). 


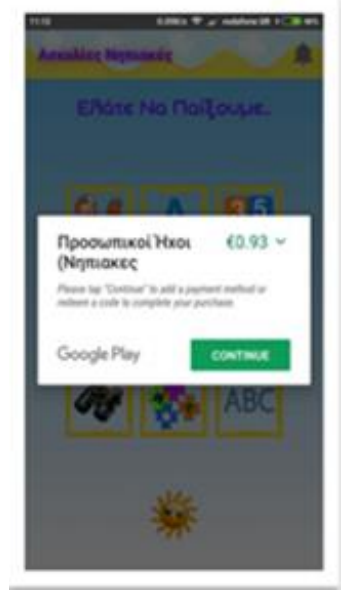

(a)

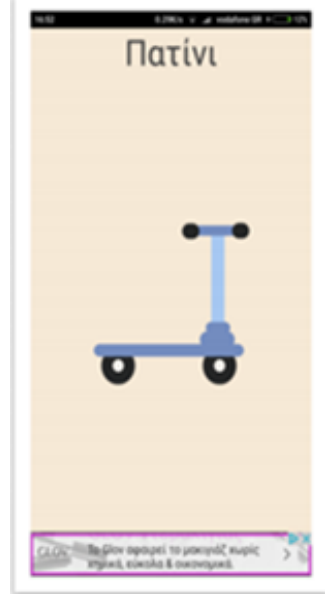

(b)

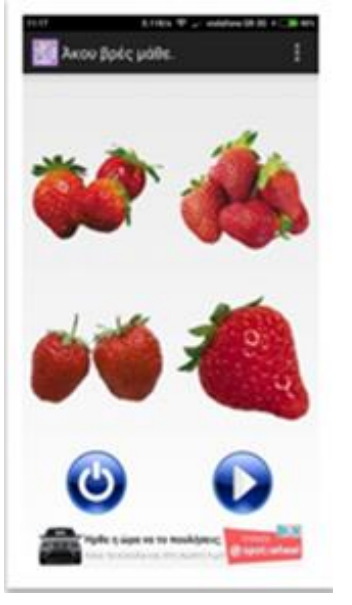

(c)

Fig. 18- shows A, B, C. Applications with embedded declarations or in-app purchases.

- Many of these applications have not accurately defined the target age group.

- None of the applications provided users with the opportunity to send and share their creations over the Internet or a virtual private network using the rich communication features of smart mobile devices (Wi-Fi, NFC, Bluetooth, etc.).

- Lack of social support in design applications, limited to white plate and storage features. These applications did not even provide the possibility of user interaction using a digital camera (Stamatios Papadakis\& Michail Kalogiannakis,2017)

\subsection{Poor quality applications:}



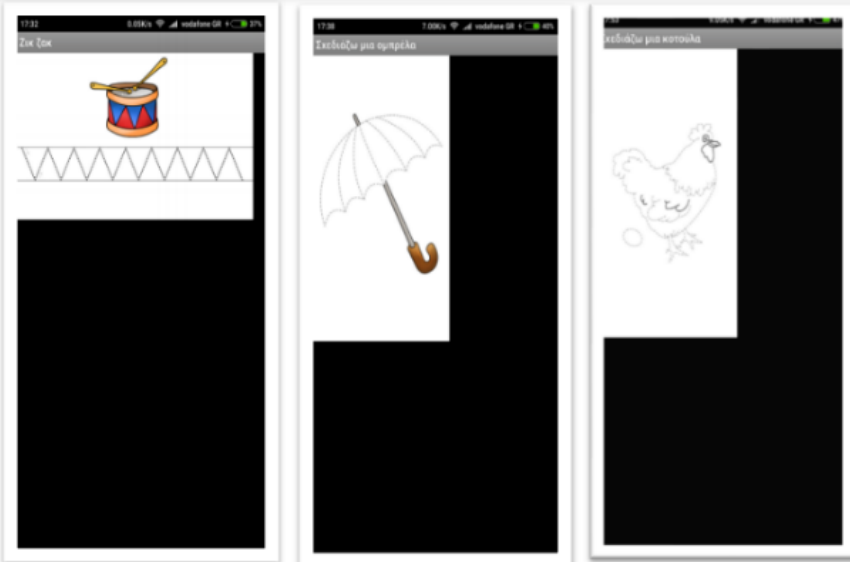

Fig. 19- shows footage from poor quality applications.

Figure 19 displays screen footage of a user interface for a poor-quality application. Buttons, controls, lists, etc., are missing. The images are very small and have not been adapted to the size of the mobile screen. The app asked learners to draw something (parachute, line, or chicken), but the images were too small to run with fingers. (Stamatios Papadakis\& Michail Kalogiannakis, 2017).

\section{CONCULSION:}

The research points to several results from them

- The use of modern technology in the education of illiterate people has become of importance associated with this era, and it has become clear that the use of modern means to educate those who are liberated from illiteracy achieve sustainability in education and increase their desire to learn about new subjects through fun educational applications and have a specific educational goal.

- Also, he found a large and important role for electronic games, for their ease of use at any time and any place, and in a fun and entertaining way for the learner to help him in continuing education and not feel bored. 


\section{INTERNATIONAL JOURNAL OF \\ DESIGN AND FASHION STUDIES}

ISSN: $2735-5365$

VOLUME 4, ISSUE 1, 2021, $160-182$.

www.egyptfuture.org/ojs/

- Diversity in the user interface of electronic games is characterized by ease of use for the learner, which relied on graphic graphics for many writings, which shows us the importance of graphic design in this area.

The research recommends several recommendations

- By inviting the official institutions concerned with literacy eradication, interest in designing educational applications suitable for individuals liberated from illiteracy.

- Inviting specialists in the field of graphic design and the concerned colleges to establish a mechanism with the relevant ministries to create designs for educational applications for literacy that contribute to easy solutions to icons, colors, and appropriate typography..etc in the implemented applications.

\section{References:}

Inayat ur-Rehman, Azra Shamim, Tamim Ahmed Khan, (2016), Mobile Based User-Centered Learning Environment for Adult Absolute Illiterates, Article, P1, PP3-4 .

Lumsden‘J., Leung, R., and Fritz‘J،‘ , June 28 - 30، (2005), Designing a Mobile Transcriber Application for Adult Literacy Education: A Case Study, National Research, Council Canada‘Institute for Information Technology, published in the Proceedings of the International Association for Development of the Information Society (IADIS) International Conference Mobile Learning, PP6-7.

Stamatios Papadakis, Michail Kalogiannakis, Nicholas Zaranis, (2017), The landscape of Android educational apps for preschoolers in Greece, A case study, Department of Preschool Education, Faculty of Education, University of Crete, Crete, Greece, PP16-17, 23-31, 19-20.

Tia Wilkinson, Walden University, (2020), Adults' Use of Mobile Learning Environments While Enrolled in General

Educational Development Classes, College of Education, Walden University, P42.

https://esrc.org.lb/article.php?id=4399\&cid=248\&catidval=0,16/5/2020, 5:15 P.M.

Jennifer Hill, Rahul Simha, (2016), Designing a Literacy-Based Mobile Application for Adult Learners, PP2 


\section{INTERNATIONAL JOURNAL OF \\ DESIGN AND FASHION STUDIES}

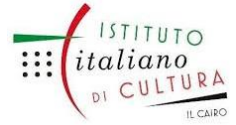

ISSN: $2735-5365$

VOLUME 4, ISSUE 1, 2021, 160 - 182.

www.egyptfuture.org/ojs/

Mohamad Adnan Al-Alaoui, Mesrob I. Ohannessian, Ghinwa F. Choueiter, September 26-28, (2007), A Pilot Project - From Illiteracy to Computer Literacy: Teaching and Learning Using Information Technology. American University of Beirut, PP2-5.

Kevin Browne, Christopher Anand، Elizabeth Gosse, , (2014), Gamification and serious game approaches for adult literacy tablet softwarePP6-9.

Received: April 2021

Accepted: June 2021 